\title{
B7-H3 Promotes the Migration and Invasion of Human Bladder Cancer Cells via the PI3K/Akt/STAT3 Signaling Pathway
}

\author{
Yuchao Li1, 2, Guoning Guo33, Jie Song2, Zhiping Cai², Jin Yang1, Zhiwen Chen", Yun Wang1, Yaqin Huang1, \\ Qiangguo Gao ${ }^{1 凶}$ \\ 1. Department of Cell Biology, Third Military Medical University, Chongqing 400038, China; \\ 2. Trainee Brigade, Third Military Medical University, Chongqing 400038, China; \\ 3. Department of Emergency, Southwest Hospital, Third Military Medical University, Chongqing 400038, China \\ 4. Urology Center, Southwest Hospital, Third Military Medical University, Chongqing 400038, China. \\ $\bowtie$ Corresponding author: gaoli@tmmu.edu.cn; Tel: +86-23-6875-2253 \\ (C) Ivyspring International Publisher. This is an open access article distributed under the terms of the Creative Commons Attribution (CC BY-NC) license \\ (https://creativecommons.org/licenses/by-nc/4.0/). See http://ivyspring.com/terms for full terms and conditions.
}

Received: 2016.09.29; Accepted: 2016.12.16; Published: 2017.02.25

\begin{abstract}
Bladder cancer is one of most common malignant cancer. Although previous studies have found abnormal expression of $\mathrm{B} 7-\mathrm{H} 3$ in human bladder cancer tissues, the exact role and molecular mechanism of B7-H3 in bladder cancer remain unknown. In this study, we first detected the expression of B7-H3 in human bladder cancer samples and cell lines, and analyzed its correlations with clinicopathological pathological parameters. Next, siRNAs or overexpression plasmids of B7-H3 were transfected into T24 or 5637 cells, and cell proliferation, apoptosis, migration and invasion were analyzed via CCK-8, colony formation, flow cytometry and transwell assays, protein expression levels were determined by western blotting. The results presented here showed B7-H3 was upregulated in bladder cancer samples compared with normal tissues, and the expression level was correlated with local invasion status. B7-H3 did not affect cell proliferation and apoptosis, but cell migration and invasion were changed through the regulation of matrix metalloproteinase (MMP) 2/9. Knockdown of B7-H3 resulted in decreased activity of the STAT3 and PI3K/Akt pathways, and the Akt served as an upstream regulator of the STAT3. Our results suggest that the overexpression of B7-H3 promotes the migration and invasion of human bladder cancer cells through the PI3K/Akt/STAT3 signaling pathway.
\end{abstract}

Key words: B7-H3, migration, invasion, human bladder cancer, PI3K/Akt/STAT3, MMP

\section{Introduction}

Bladder cancer, the second most common form of malignant genitourinary cancer, has been estimated to occur in 76,960 new cases and result in 16,390 deaths in the United States in 2016. Despite advances in surgical techniques and improvements in systemic chemotherapies, there has been no change in the 5-year survival rate of bladder cancer from 1987-1989 to 2005-2011[1]. Therefore, new therapies for bladder cancer need to be developed as quickly as possible.

Recently, novel immunotherapeutic manipulations for cancer have emerged. These treatment modalities include monoclonal antibodies, cancer vaccines and cytokine therapies[2]. Bacillus Calmette-Guérin (BCG) was first reported as an immunotherapeutic agent for the treatment of non-muscle invasive bladder cancer, whereas checkpoint inhibitors are leading the way for metastatic bladder cancer[3]. Members of the B7 superfamily, such as cytotoxic T-lymphocyteassociated antigen 4 (CTLA-4), programmed cell death 1 (PD-1) and programmed cell death ligand 1 (PD-L1), represent negative co-stimulators that inhibit $\mathrm{T}$ cell function, and inhibitors of these factors hold promise in the treatment of bladder cancer [4-6]. However, objective response rates have been higher for patients with high expression of PD-L1 / PD-1 than for those with low expression of these factors. Thus, a new checkpoint that has high expression in bladder 
cancer cells may serve as a novel target for therapy.

B7-H3 (B7 homologue 3, CD276), a member of the B7/CD28 superfamily, is widely expressed at the mRNA level in most organs, but its protein expression is relatively restricted and low $[7,8]$. The extracellular architecture of B7-H3 is characterized by a 2IgB7-H3 or 4IgB7-H3 domain that contains conserved cysteine residues. The predominant isoform of $\mathrm{B} 7-\mathrm{H} 3$ in human tissues and cell lines is 4IgB7-H3 rather than 2IgB7-H3 [9].

The functional role of B7-H3 in tumor immunity is controversial. The contrasting roles of $\mathrm{B} 7-\mathrm{H} 3$ are likely attributable to the activities of multiple receptors on different cells. Although triggering receptor expressed on myeloid cells (TREM)-like transcript 2 (TLT-2) was demonstrated as a costimulatory receptor of murine $\mathrm{B} 7-\mathrm{H} 3$, no evidence for the existence of human TLT-2 has been found [10]. The lack of identification of human B7-H3 receptors hinders further immunological studies.

There conflicting evidence regarding a non-immunological role of B7-H3 in cancer progression. Reports demonstrate that overexpression of B7-H3 correlates with disease severity and poor outcome in many cancer types [11], but it has also been associated with favorable outcomes in pancreatic cancer and gastric cancer $[12,13]$. It was reported that the expression of B7-H3 was potentially increased in urothelial cell carcinoma samples compared with non-tumor urothelial samples [14-16] and that MGA271, the engineered anti-B7-H3 mAb, exhibited potent in vivo antitumor activity against bladder cell carcinoma xenografts [17]. However, the functions and molecular mechanisms of B7-H3 in bladder cancer are poorly understood.

In this study, we have investigated the expression, function and molecular mechanisms of B7-H3 in bladder cancer. Our data show that overexpression of B7-H3 in bladder cancer cells promotes cell migration and invasion via the phosphatidylinositol 3-kinase (PI3K)/Akt/STAT3 signaling pathway.

\section{Materials and Methods}

\section{Patients and tissue specimens}

Samples of bladder urothelial carcinoma tissue and adjacent normal tissue were obtained from 45 patients (25 males and 20 females) via transurethral bladder tumor resection and radical cystectomy at Southwest Hospital, Third Military Medical University. The mean age of the patients was 65 years (ranging from 35 to 79 years). Tumor tissues were examined by a pathologist, and tumor grade of urothelial carcinoma was classified as low or high according to the WHO criteria (2004), and the tumor stage was assigned as low (superficial, Ta-T1) and high (muscle invasive, T2-T4) according to the American Joint Committee on Cancer tumor node metastasis (TNM) staging system (2002). This research was approved by the ethics board of the Third Military Medical University.

\section{Cell culture and reagents}

The human bladder cancer cell lines RT4, 5637, J82, and T24 were obtained from ATCC (Rockville, $\mathrm{MD}$, USA) and maintained according to the manufacturer's instructions. The medium was supplemented with $50 \mu \mathrm{M}$ LY294002 for inhibition of PI3K or $3 \mu \mathrm{M}$ WP1066 (Medchem Express, USA) for inhibition of Stat3, with DMSO as a control for $24 \mathrm{~h}$.

\section{Immunohistochemistry and immunofluorescence assays}

Immunohistochemistry was performed according to previously described procedures [18]. Staining for B7-H3 was conducted using a goat anti-human 4IgB7-H3 antibody (5 $\mu \mathrm{g} / \mathrm{ml}$, R\&D Systems, USA). For cultured cells, a Cy3-labeled donkey anti-goat antibody (1:200) was used for immunofluorescence staining, followed by nuclear staining using DAPI ( $5 \mu \mathrm{g} / \mathrm{ml})$.

\section{Cell transfections}

According to the mRNA sequence of 4IgB7-H3 in GenBank (Gene ID: 80381), three siRNAs were designed. The specific siRNAs and the negative control siRNA (siNC) were synthesized by Shanghai GenePharma Company. The siRNA sequences are shown in Table 1.

To upregulate B7-H3 expression in 5637 cells, a pCMV3 vector encoding full-length B7-H3 (pCMV3-B7-H3) plasmid was designed and purchased from Sino Biological Inc. (Beijing, China), pCMV3 was used as negative control.

Cells were seeded in 6-well plates at $1 \times 10^{5}$ cells $/ \mathrm{ml}$ and transfected for 5 hours according to the manufacturer's transfection protocol. Then, the cells were analyzed via quantitative RT-PCR (Q-PCR) and western blotting at the indicated time points.

Table 1. The siRNA sequences used for B7-H3 knockdown.

\begin{tabular}{ll}
\hline siRNA & Primer sequence \\
\hline siB7-H3a (B7-H3-homo -435) & F: 5'-GCAGCUGACAGAUACCAAATT-3' \\
& R: 5'-UUUGGUAUCUGUCAGCUGCTT-3' \\
siB7-H3b (B7-H3-homo -996) & F: 5'-GCUGUCUGUCUGUCUCAUUTT-3' \\
& R: 5'-AAUGAGACAGACAGACAGCTT-3' \\
siB7-H3c (B7-H3-homo -1041) & F: 5'-GUGCUGGAGAAAGAUCAAATT-3' \\
& R: 5'-UUUGAUCUUUCUCCAGCACTT-3' \\
siNC (Negative control) & F: 5'-UUCUCCGAACGUGUCACGUTT-3' \\
& R: 5'-ACGUGACACGUUCGGAGAATT-3' \\
\hline
\end{tabular}




\section{Q-PCR and western blotting}

Q-PCR and western blotting were performed as described in a published article [19].

The primer sequences for Q-PCR are shown in Table 2.

Table 2. The primers used for target genes.

\begin{tabular}{lll}
\hline Gene symbol & Primer sequence & $\begin{array}{l}\text { Product } \\
\text { length (bp) }\end{array}$ \\
\hline B7-H3 & F: 5'-GGACATAGCCCCTCGCCAC-3' & 162 \\
& R: 5'-CTCGGTGTCCCCAAAGCATT-3' & \\
MMP2 & F: 5'-TACAGGATCATTGGCTACACACC-3' & 90 \\
& R: 5'-GGTCACATCG CTCCAGACT-3' & \\
MMP9 & F: 5'-TGTACCGCTATGGTTACACTCG-3' & 97 \\
& R: 5'-GGCAGGGACAGTTGCTTCT-3' & \\
GAPDH & F: 5'-GGAGCGAGATCCCTCCAAAAT-3' & 197 \\
& R: 5'-GGCTGTTGTCATACTTCTCATGG-3' & \\
\hline
\end{tabular}

The primary antibodies included antibodies against B7-H3, PI3K, p-PI3K, Akt, p-Akt, STAT3, p-STAT3 (Cell Signaling Technology, USA), Ki67 (Santa Cruz biotechnology, USA), matrix metalloproteinase (MMP) 2, and MMP9 (GeneTex, USA), GAPDH.

\section{Proliferation assay}

After transfection for $48 \mathrm{~h}, 1000$ cells per well were seeded in 96-well plates, and cell proliferation was measured using a Cell Counting Kit-8 (CCK-8; Dojindo, Kumamoto, Japan) according to the manufacturer's instructions. Proliferation rates were determined at $24,48,72$ and $96 \mathrm{~h}$ after transfection by measuring the absorbance at $450 \mathrm{~nm}$ in a microplate reader (Bio-Rad, USA).

\section{Clone formation assay}

After transfection for $48 \mathrm{~h}$, the treated cells were seeded as single cells in 6-well plates (1000 cells per well), and the cells were cultured for 14 days until large colonies were visible. The colonies were fixed with $4 \%$ paraformaldehyde and stained with crystal violet dye for $5 \mathrm{~min}$. The dishes were photographed, and the number of colonies was counted under a phase-contrast microscope.

\section{In vitro cell migration and invasion assay}

Cell migration and invasion were measured using transwell chambers (Corning, USA) containing 24-well inserts with $8 \mu \mathrm{m}$ pores in the presence or absence of Matrigel (BD Biosciences, USA) according to the manufacturer's protocol. At $48 \mathrm{~h}$ after transfection, T24 cells were incubated for an additional $4 \mathrm{~h}$ for migration or $24 \mathrm{~h}$ for invasion, the 5637 cells were cultured for $6 \mathrm{~h}$ for migration or $36 \mathrm{~h}$ for invasion. Then, the cells in the upper chamber were removed, and the remaining cells were fixed in
4\% paraformaldehyde and stained with crystal violet solution. Cells were quantified in five randomly selected fields for each membrane, and the average cell count for three independent membranes was defined as the migration or invasion index.

\section{Cell apoptosis assay}

The effects of B7-H3 on cell apoptosis were detected by Annexin V/ propidium iodide (PI) double staining. T24 or 5637 cells were harvested at 48 $\mathrm{h}$ post-transfection by trypsinization (without EDTA), and resuspended in $1 \times$ binding buffer at $1 \times 10^{6}$ cells $/ \mathrm{ml}$. After double staining with AnnexinV-APC and PI (eBioscience, USA), cells were collected and analyzed using an Accuri C6 flow cytometry (BD, USA). Experiments were performed for three independent times.

\section{Statistical analysis}

For continuous variables, two-tailed Student's $\mathrm{t}$-tests were performed for comparisons between the experimental and control groups, data were summarized as the mean $\pm \mathrm{SD}$. One-way analysis of variance (ANOVA) followed by Scheffe's post hoc test was performed to compare the differences between multiple groups. To evaluate the correlation between B7-H3 expression and clinicopathological categorical variables, the Chi-square test and Fisher's exact test were performed to compare the ratios. Statistical analysis was performed using SPSS 11.0 software, and a value of $P<0.05$ was considered significant.

\section{Results}

\section{The expression of B7-H3 in human bladder cancer tissues}

Previous articles have reported that B7-H3 is expressed in bladder cancer samples, but the details of its expression at different stages have not been described [14-16]. To identify the expression of B7-H3 in human bladder cancer, we detected the expression of B7-H3 in 45 primary bladder cancer samples and corresponding adjacent normal tissues via Q-PCR and immunohistochemistry. The mRNA level of B7-H3 was significantly higher in tumor tissues than in adjacent normal tissues, but there was no apparent difference in B7-H3 mRNA expression between tumor tissues according to tumor grade or stage (Fig. 1A). Immunohistochemical staining revealed a significantly greater percentage of bladder urothelial carcinoma samples expressing B7-H3 than adjacent normal tissue samples ( $\mathrm{P}<0.001$, Table 3$)$, the intensity of B7-H3 expression was markedly increased in malignant tissue compared to normal tissue, and B7-H3 expression was localized to the entire urothelium (Fig. 1B). Based on multivariate regression 
analysis, the results showed that the expression level of B7-H3 was correlated with local invasion status (Ta-T1 vs.T2-T4, P=0.039. Table 4), but it was not associated with the age, gender and the tumor grade. These results implied that the overexpression of B7-H3 may be related to the invasion of bladder cancer.

Table 3. The frequency of $\mathrm{B} 7-\mathrm{H} 3$ expression in bladder urothelial carcinoma tissues and adjacent normal tissues.

\begin{tabular}{lllll}
\hline Classification & Case & B7-H3(-) & B7-H3(+) & $P$ value \\
\hline Normal tissue & 45 & 20 & 25 & $P<0.001$ \\
$\begin{array}{l}\text { Carcinoma } \\
\text { tissue }\end{array}$ & 45 & 3 & 42 & \\
\hline
\end{tabular}

Table 4. Association of B7-H3 expression with clinicopathological characteristics of the bladder urothelial carcinoma tissues.

\begin{tabular}{|c|c|c|c|c|c|c|}
\hline \multirow[t]{2}{*}{ Parameters } & \multirow[t]{2}{*}{ Case } & \multicolumn{4}{|c|}{ B7-H3 expression } & \multirow{2}{*}{$\begin{array}{l}P \\
\text { values }\end{array}$} \\
\hline & & Negative & Low & Moderate & Intense & \\
\hline \multicolumn{7}{|l|}{ Age (years) } \\
\hline Mean (SD) & 45 & $55.7 \pm 5.8$ & $66.2 \pm 8.3$ & $58.2 \pm 11.8$ & $60.9 \pm 14.3$ & 0.544 \\
\hline \multicolumn{7}{|l|}{ Gender } \\
\hline Male & 25 & 2 & 4 & 12 & 7 & 0.457 \\
\hline Female & 20 & 1 & 1 & 8 & 10 & \\
\hline \multicolumn{7}{|l|}{ Tumor grade } \\
\hline Low grade & 20 & 1 & 2 & 11 & 6 & 0.689 \\
\hline High grade & 25 & 2 & 3 & 9 & 11 & \\
\hline \multicolumn{7}{|l|}{ Tumor stage } \\
\hline Low (Ta-T1) & 20 & 1 & 1 & 13 & 5 & $0.039^{*}$ \\
\hline High (T2-T4) & 25 & 2 & 5 & 6 & 12 & \\
\hline
\end{tabular}

\section{The expression of $\mathrm{B} 7-\mathrm{H} 3$ in bladder cancer cell lines}

To select appropriate bladder cancer cell lines to study the function of B7-H3, we selected RT4, 5637, T24 and J82 cells to examine B7-H3 expression. The results showed higher mRNA and protein expression levels of B7-H3 in T24 cells than in RT4, 5637 and J82 cells, while the B7-H3 expression in 5637 cells was lower (Fig. 1C and D). Immunofluorescence showed positive B7-H3 staining in the cell membrane and cytoplasm. Additionally, the expression level of B7-H3 was higher in T24 cells than in the other cell types examined (Fig. 1E). Thus, we selected T24 and 5637 cells to study the function of B7-H3.

\section{Inhibition or overexpression of B7-H3 in bladder cancer cells}

To select the most efficient siRNA for further experiments, mRNA and protein expression of $\mathrm{B} 7-\mathrm{H} 3$ was analyzed after siRNA transfection. The expression level was markedly decreased $24 \mathrm{~h}$ after siRNA transfection compared to negative control treatment based on Q-PCR (Fig. 2A). A similar decrease was found in protein expression in cells $48 \mathrm{~h}$ after B7-H3 knockdown based on western blotting (Fig. 2B). These results indicate that the down-regulation of B7-H3 via RNA interference was specific and efficient. We selected siB7-H3a for subsequent assays because this siRNA exhibited the greatest silencing effect among all siRNAs tested. Also the overexpression vector increased the mRNA and protein expression of B7-H3 in 5637 cells (Fig. 3A, B).
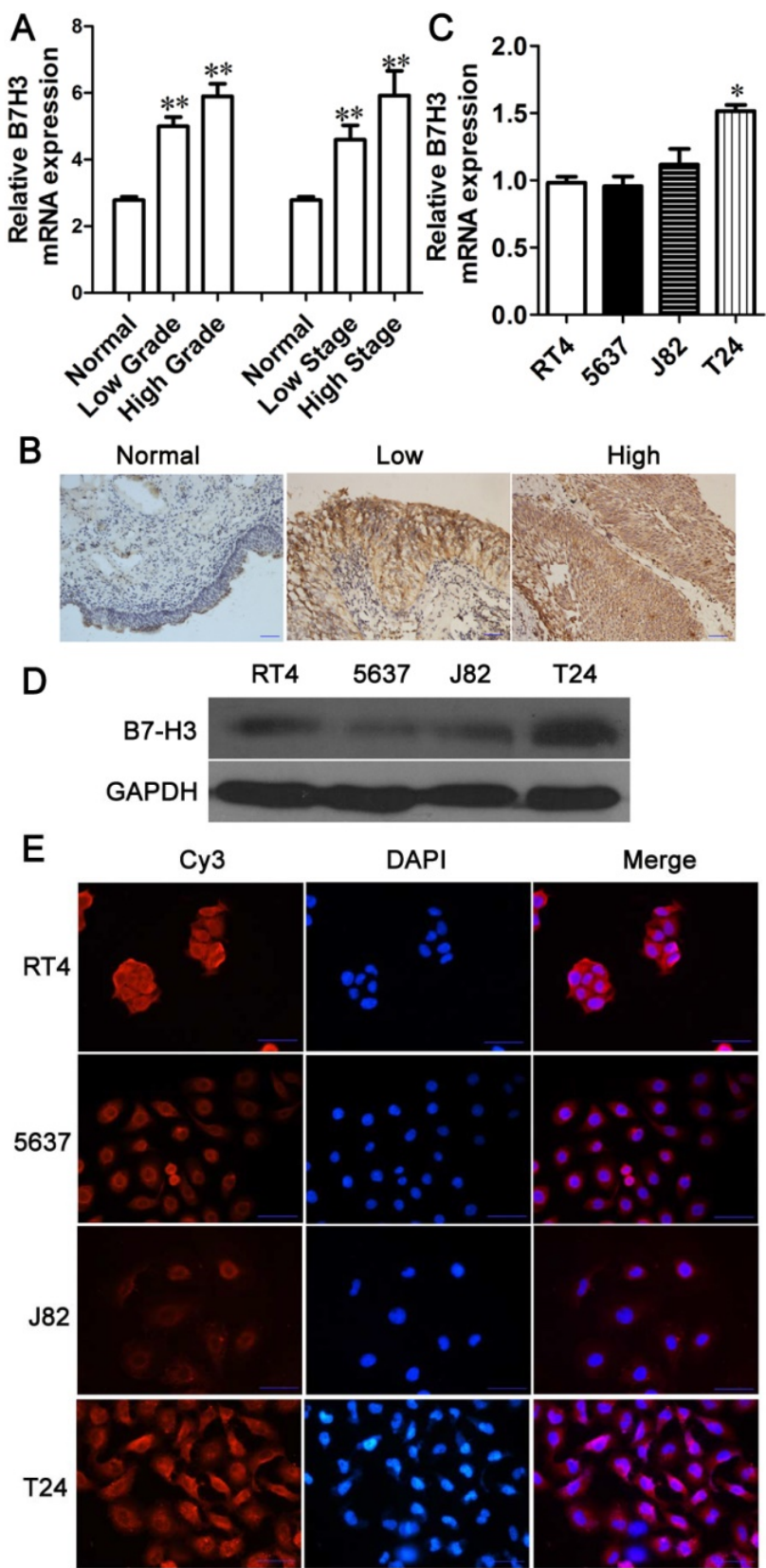

Fig. 1. Upregulated expression of $B 7-\mathrm{H} 3$ in human bladder cancer tissues and cell lines. The mRNA (A) and protein (B) expression levels of B7-H3 in adjacent normal tissues and human bladder cancer tissues (A, B) and different cell lines (C, D) were determined via Q-PCR, immunohistochemistry or western blotting. Immunofluorescence for B7- $\mathrm{H} 3$ expression in different cell lines was examined using the Cy3, DAPI, and merged channels (E). (Bar $=50 \mu \mathrm{m}, * P<0.05$, $* * P<0.01)$. 

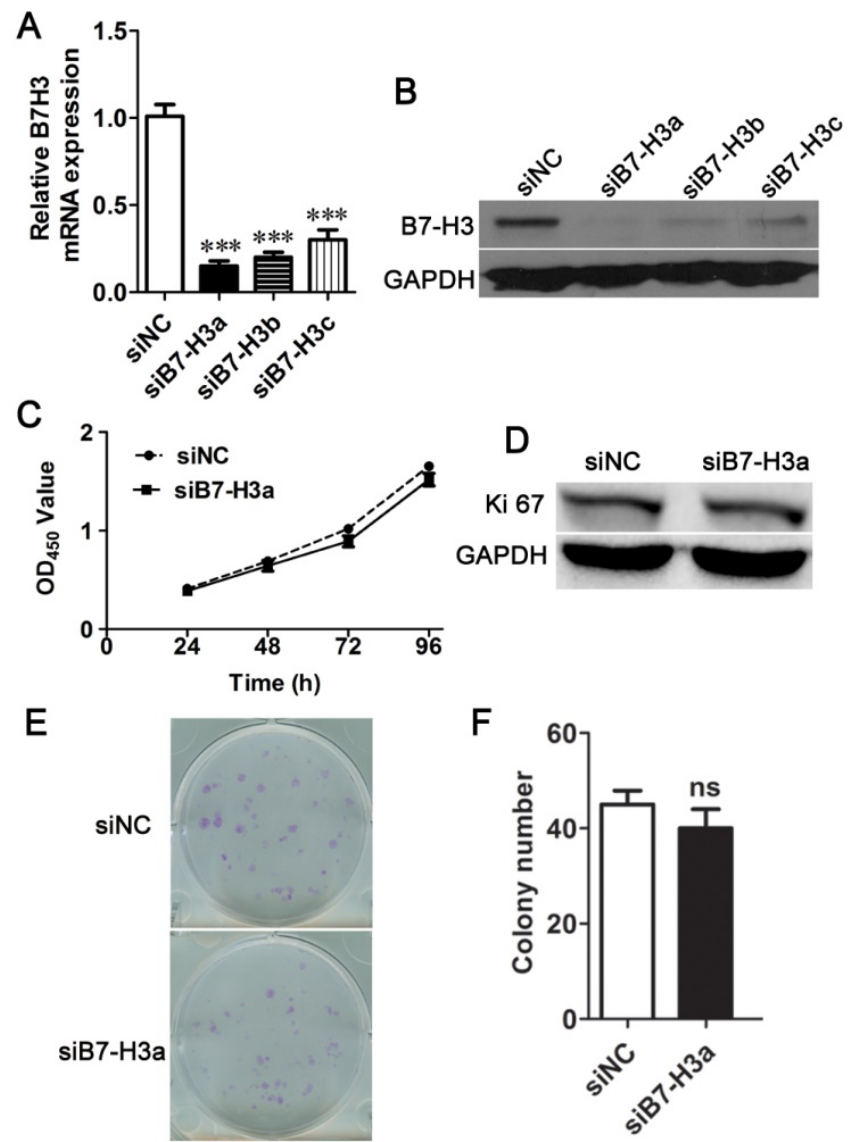

Fig. 2. No significant difference in cell proliferation was detected between siNC- and siB7-H3-treated T24 cells. The relative mRNA (A) and protein (B) B7-H3 expression levels after RNA interference were determined via Q-PCR or western blotting, respectively. (C) The proliferation of siNC- or siB7-H3-treated cells was measured via CCK-8 assays. (D) Western blotting analysis for the expression of Ki67 after B7-H3 knockdown. T24 cells transfected with siNC or siB7-H3 were allowed to form colonies $(E)$, and the number of colonies was counted $(F) .(* * * P<0.001)$.

\section{B7-H3 does not control the proliferation of bladder cancer cells}

To characterize the role of $\mathrm{B} 7-\mathrm{H} 3$ in the growth of bladder cancer, T24 cells treated with B7-H3 siRNA were examined by the CCK- 8 assay. There was no significant difference in cell proliferation between the control and experimental groups (Fig. 2C). The expression of Ki67, marker of cell proliferation, was examined by western blotting, and the results indicated no change in its expression (Fig. 2D). A colony formation assay showed no difference in the cell colony count between the B7-H3-silenced cells and the control cells (Fig. 2E and F). Consistent with the above assay, the proliferation of 5637 cells detected by using the CCK-8, Ki67 and cell colony assay showed a similar results (Fig. 3C, D, E and F).These data implied that B7-H3 is not associated with the proliferation of bladder cancer cells.

A
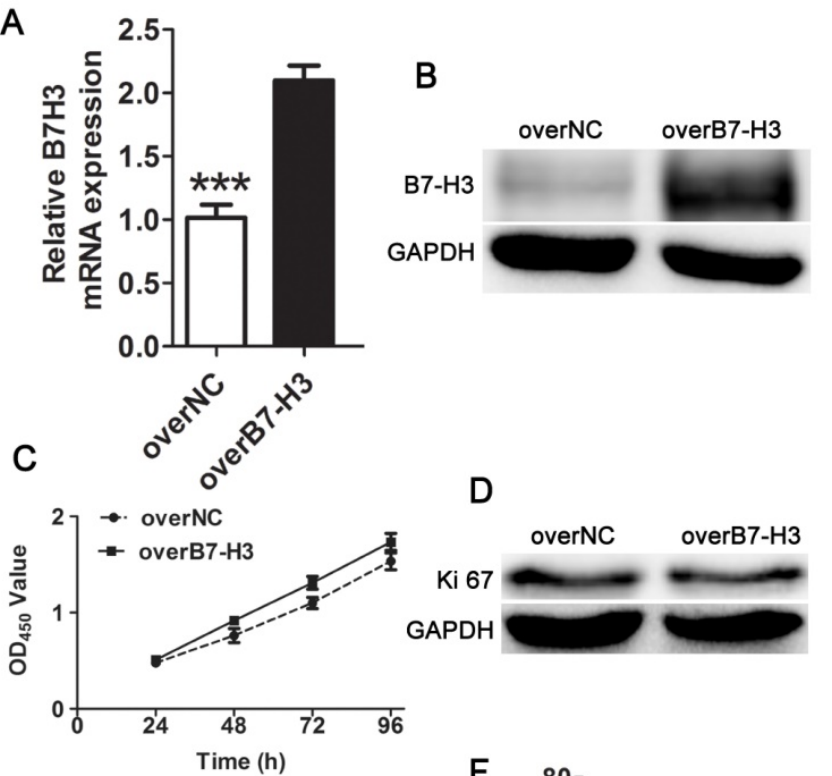

E

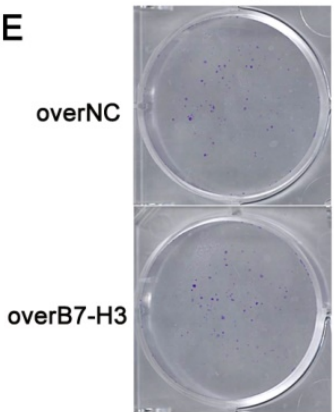

F

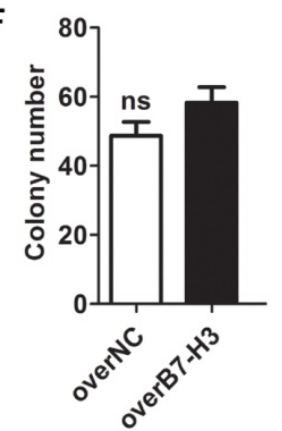

Fig. 3. B7-H3 does not control cell proliferation of 5637 cells. The relative mRNA (A) and protein (B) B7-H3 expression levels in 5637 cells were evaluated using Q-PCR or western blotting. Cell proliferation was measured by CCK-8 assays (C), Ki67 (D), and clone formation assay (E, F).

\section{B7-H3 does not regulate cell apoptosis in bladder cancer cells}

To document the effect of B7-H3 on cell apoptosis in bladder cancer cells, flow cytometry was performed to evaluate the apoptosis by Annexin V kit. After $48 \mathrm{~h}$, Annexin V/PI staining showed that the percentage of apoptotic cells has no obvious difference between the treated groups and control groups (Fig. 4A, B). These results indicated that B7-H3 is not related to cell apoptosis.

\section{B7-H3 regulates the migration and invasion of bladder cancer cells}

To determine whether B7-H3 modulates tumor migration and invasion, transwell assays were performed. siRNA-mediated silencing of B7-H3 significantly decreased the migration of T24 cells (Fig. $5 \mathrm{~A}, \mathrm{C})$, in contrast, the ability of cell migration was enhanced by the B7-H3 overexpression vector in 5637 cells (Fig. 5E, G). The number of migrated cells was decreased or increased by approximately $53 \%$ or $51 \%$ 
in the experimental cells compared with the control cells. Using a Matrigel-coated Boyden chamber assay, we found that the number of invaded cells had decreased by up to $50 \%$ following B7-H3 knockdown after $48 \mathrm{~h}$ (Fig. 5B, D), while the invaded cells had increased to $55 \%$ by the overexpression of B7-H3 (Fig. $5 \mathrm{~F}, \mathrm{H})$. These data suggested that $\mathrm{B} 7-\mathrm{H} 3$ plays an important role in cell migration and invasion.

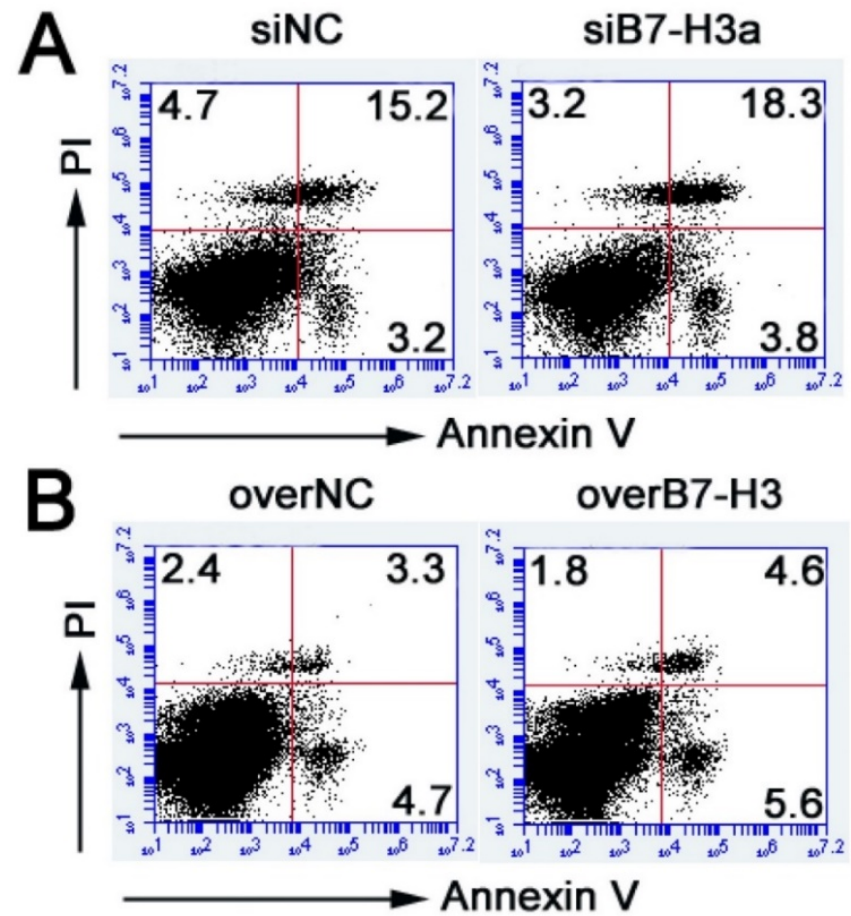

Fig. 4. Role of B7-H3 in cell apoptosis of bladder cancer cells. Annexin V-APC/PI analysis showed no obvious differences between the treated groups of knockdown B7-H3 in T24 cells (A) or overexpression of B7-H3 in 5637 cells (B) and the control groups.
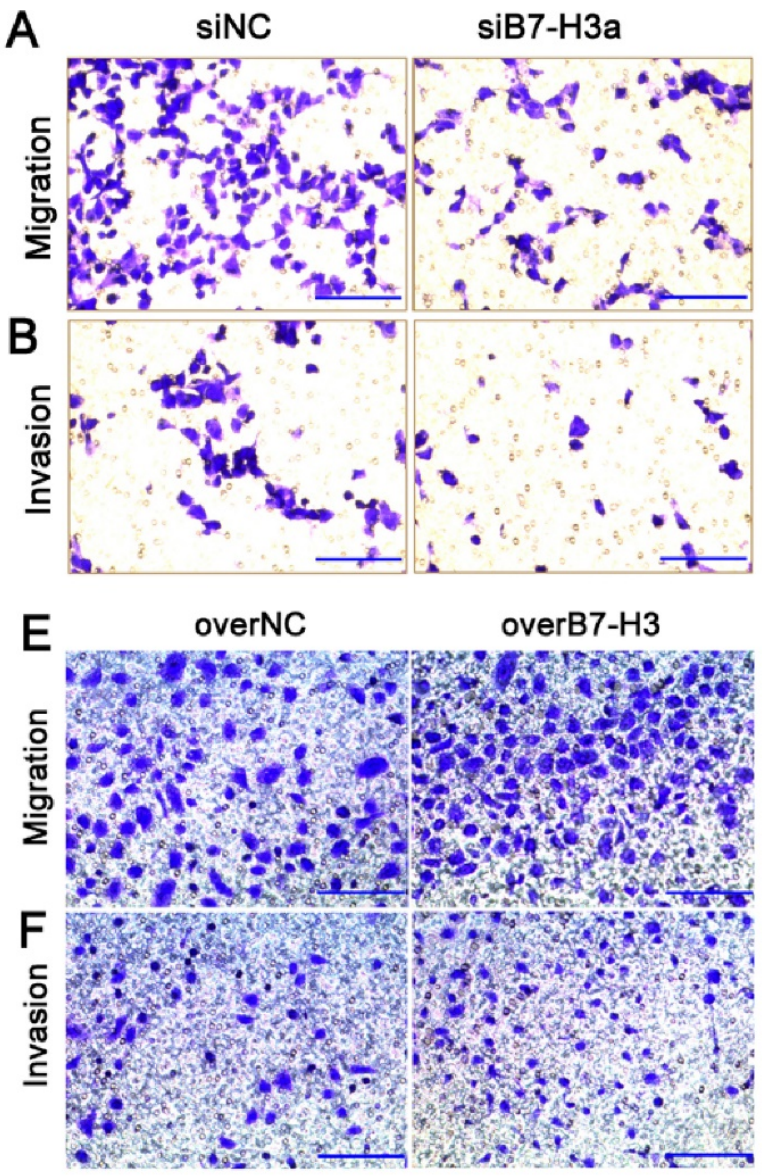
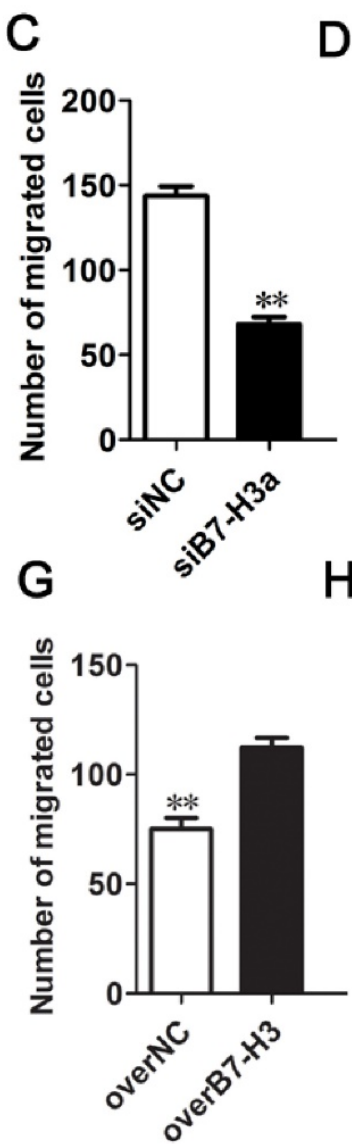

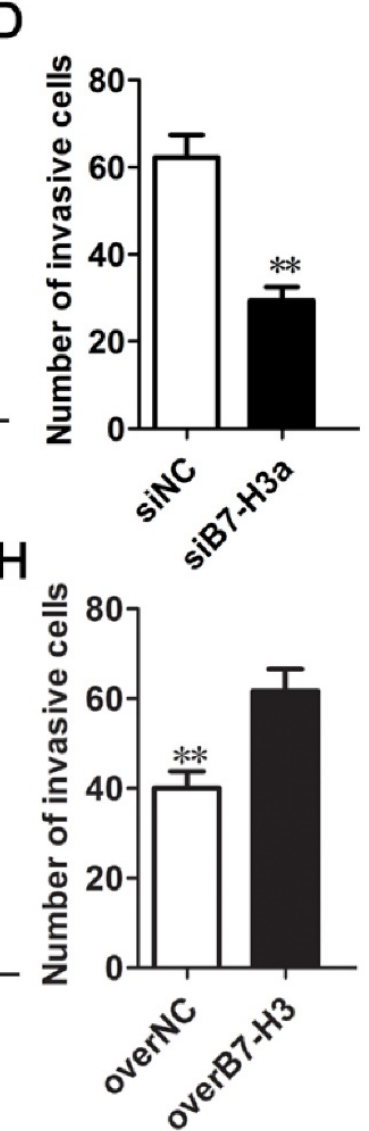

Fig. 5. Effects of $B 7-\mathrm{H} 3$ on the migration and invasion of bladder cancer cells. T24 cells were transfected with siNC or siB7-H3, and the cell migration (A) and invasion (B) were evaluated via transwell assays, the numbers of migrated $(C)$ and invaded cells $(D)$ were counted. The ability of migration $(E)$ and invasion (F) of 5637 cells transfected with overNC or overB7-H3 was tested by transwell assays, the numbers of migrated $(\mathrm{G})$ and invaded cells $(\mathrm{H})$ were counted. $(\mathrm{Bar}=50 \mu \mathrm{m}, * * \mathrm{P}<0.01)$. 


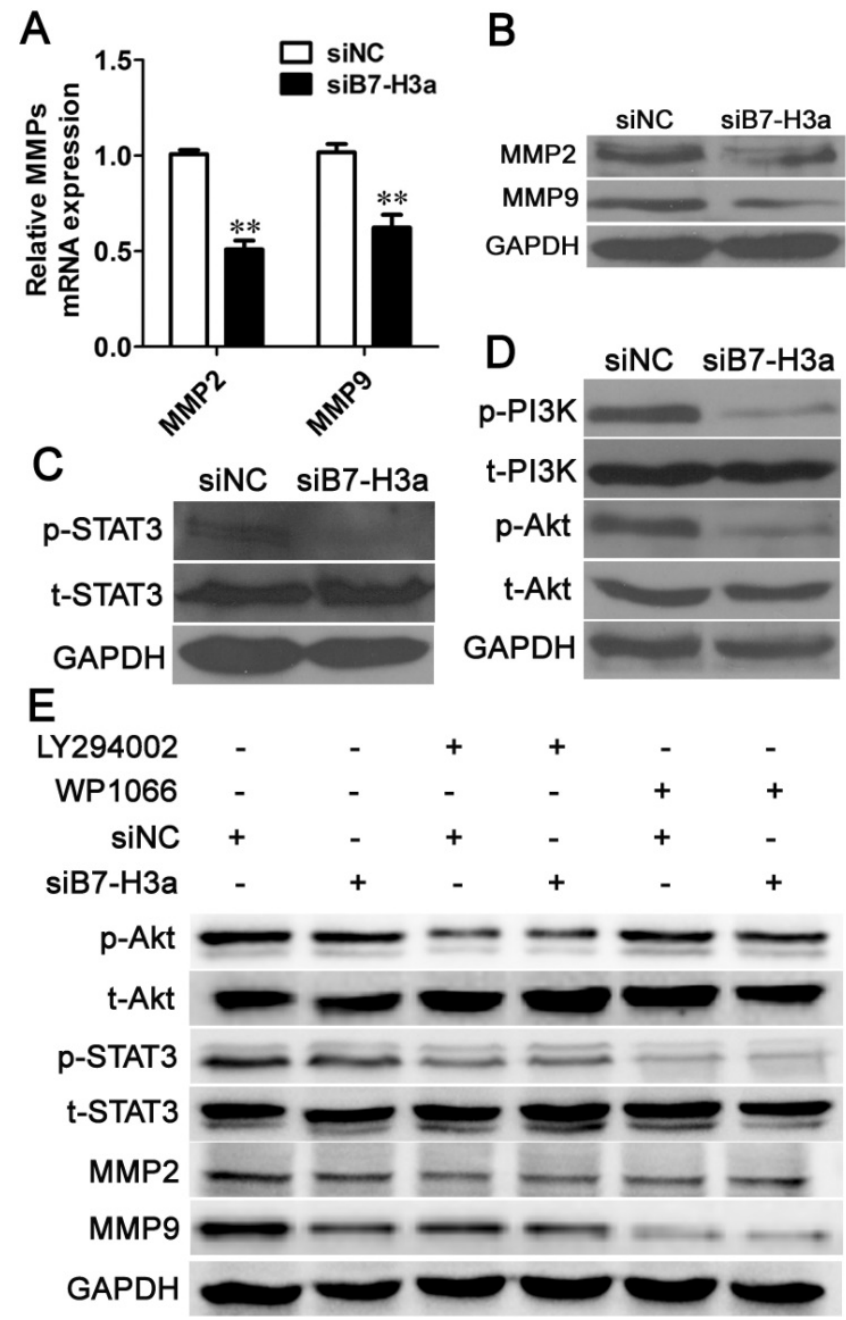

Fig. 6. Knockdown of $B 7-\mathrm{H}_{3}$ inhibits cell migration and invasion via the $\mathrm{PI3K} / \mathrm{Akt} / \mathrm{STAT} 3$ signaling pathway. Knockdown of B7-H3 decreased the mRNA (A) and protein (B) expression of MMP2 and MMP9 based on Q-PCR and western blotting, respectively. The protein expression levels of p-STAT3, total (t)-STAT3 (C), $\mathrm{p}-\mathrm{PI} 3 \mathrm{~K}, \mathrm{t}-\mathrm{PI} 3 \mathrm{~K}, \mathrm{p}-\mathrm{Akt}$ and t-Akt (D) $48 \mathrm{~h}$ after RNA interference using siNC or siB7-H3 were determined via western blotting. (E) The protein expression levels of p-Akt, t-Akt, P-STAT3, t-STAT3, MMP2 and MMP9 were determined via western blotting $48 \mathrm{~h}$ after knockdown of $\mathrm{B} 7-\mathrm{H} 3$ and blockade of the PI3K/Akt pathway using LY294002 or the STAT3 pathway using WP1066.

\section{B7-H3 regulates the expression of MMP2 and MMP9}

Degradation of the basement membrane and the extracellular matrix (ECM) is vital for the invasion of tumor cells. MMP2 and MMP9 can degrade type IV collagen in the basement membrane and can further degrade the collagenous and non-collagenous components of the ECM [20]. Thus, MMP2 and MMP9 are regarded as important markers of cancer cell invasion. We determined the expression levels of MMP2 and MMP9 via Q-PCR and western blotting. As shown in Fig. 6A, siB7-H3 transfection significantly decreased the MMP2 and MMP9 mRNA levels, and western blotting analysis showed that siB7-H3 transfection decreased the expression of both
MMP2 and MMP9 in T24 cells (Fig. 6B). These results indicated that $\mathrm{B} 7-\mathrm{H} 3$ inhibited cell migration and invasion by decreasing the expression of MMP2 and MMP9.

\section{B7-H3 regulates cell migration and invasion via the PI3K/Akt/STAT3 signaling pathway}

As B7-H3 regulated the expression of MMP2 and MMP9, we were interested in the molecular mechanism underlying this activity. Previous studies have shown that B7-H3 stimulates invasion of hepatocellular carcinoma and melanoma cells via the JAK2/STAT3/Slug or STAT3 signaling pathways, respectively [21, 22]. We also found that siB7-H3 transfection decreased the expression level of p-STAT3 in bladder cancer cells (Fig. 6C). Many articles have reported that the PI3K / Akt signaling pathway is involved in invasion of cancer cells, such as breast cancer, gastric adenocarcinoma, and lung cancer cells [23-25]; However, no report has described the relationship of B7-H3 to this pathway. Thus, we performed experiments to explore whether B7-H3 could affect this pathway in bladder cancer. Western blotting revealed that the expression levels of p-PI3K and p-Akt were suppressed when $\mathrm{B} 7-\mathrm{H} 3$ was downregulated (Fig. 6D).

To explore whether there was cross-talk between the STAT3 and PI3K/Akt pathways, we performed experiments using the PI3K inhibitor LY294002 and the STAT3 inhibitor WP1066. We observed that the expression of both p-Akt and p-STAT3 was effectively suppressed upon addition of LY294002. However, WP1066 only abolished the activation of STAT3 but not p-Akt. These results implied that the activation of STAT3 is dependent on the PI3K/Akt pathway. As B7-H3 regulated the expression of p-Akt and p-Stat3, which indicated that B7-H3 may be an upstream regulator of PI3K/Akt/STAT3. Furthermore, siB7-H3 transfection significantly decreased the expression of MMP2 and MMP9 cooperated with LY294002 or WP1066 (Fig. 6E). These data strongly suggest that the PI3K/Akt/STAT3 signaling pathway regulates B7-H3-mediated cell migration and invasion.

\section{Discussion}

B7-H3 displays distinct expression profiles and functions between different organs, therefore, appropriate research methods such as multi-center studies of large numbers of patients would be required to draw more clinically relevant conclusions. It was reported that B7-H3 mRNA is expressed in normal bladder tissue [7]. In the present study, we observed restricted, low expression of $\mathrm{B} 7-\mathrm{H} 3$ in adjacent normal tissues but high levels of B7-H3 expression in bladder cancer tissue based on Q-PCR 
and immunohistochemistry, and our results are consistent with previous findings [14-16]. A correlation was observed between overexpression of B7-H3 and tumor stages (invading depth), for the expression level of B7-H3 was higher in T2-T4 tumors than in Ta-T1 tumors, then analysis of B7-H3 expression may be necessary for the prediction of bladder cancer prognosis. Previous articles showed that the soluble B7-H3 level in serum correlated with the clinical stage of clear cell renal cell carcinoma, hepatocellular carcinoma and non-small cell lung cancer [26-28]. Further investigation of the B7-H3 level in bladder cancer tissues or serums may reveal B7-H3 as a sensitive biomarker for cancer diagnosis.

Tumor metastasis is regarded as the main cause of cancer-related deaths. Breakdown of the basement membrane and invasion of tumor cells are regarded as key steps in tumor metastasis. Previous reports revealed that MMPs modulate multiple tumor-supporting cellular processes. In our study, knockdown of B7-H3 decreased the mRNA and protein expression of MMP2 and MMP9. These results suggest that $\mathrm{B} 7-\mathrm{H} 3$ is involved in the transcriptional regulation of MMP2 and MMP9. Post-transcriptional mechanisms are central to the control of MMP activity because MMPs are secreted in inactive zymogens and are activated by cleavage of their N-terminal prodomain. Currently, the formation of actin-rich invasive protrusions is regarded as a key step in cancer cell invasion, and MMP2 and MMP9 are enriched at invadopodia [29]. In general, the modulation of invasion-related molecules further supports the function of $\mathrm{B} 7-\mathrm{H} 3$ in bladder cancer cells. In addition, we investigated the cell proliferation and apoptosis in bladder cancer cells, unfortunately, there were no obvious differences between the treated groups and control groups. These results indicate that B7-H3 may play important roles in the migration and invasion, not affect the proliferation and apoptosis.

It was reported that $42 \%$ of all bladder cancer patients harbor mutations in the PI3K/Akt/mTOR pathway [30] and that the PI3K/Akt pathway can regulate bladder cancer cell invasion [31]. However, no report has described the relationship between B7-H3 and this pathway. STAT3 is an important transcription factor that can translocate to the nucleus to regulate the expression of essential pro-invasive factors, such as MMPs, HSP70 and HSP90. B7-H3 can promote cell invasion via the STAT3 signaling pathway [21, 22]. In this study, we detected cross-talk between the PI3K/Akt and STAT3 pathways in which Akt served as an upstream regulator of STAT3. Although we demonstrated that $\mathrm{B} 7-\mathrm{H} 3$ can regulate the PI3K/Akt/STAT3 signaling pathway, further investigation is required to determine whether other signaling pathways are also involved in the function of B7-H3. Additionally, in vivo experiments should be performed in future studies to confirm the roles of B7-H3.

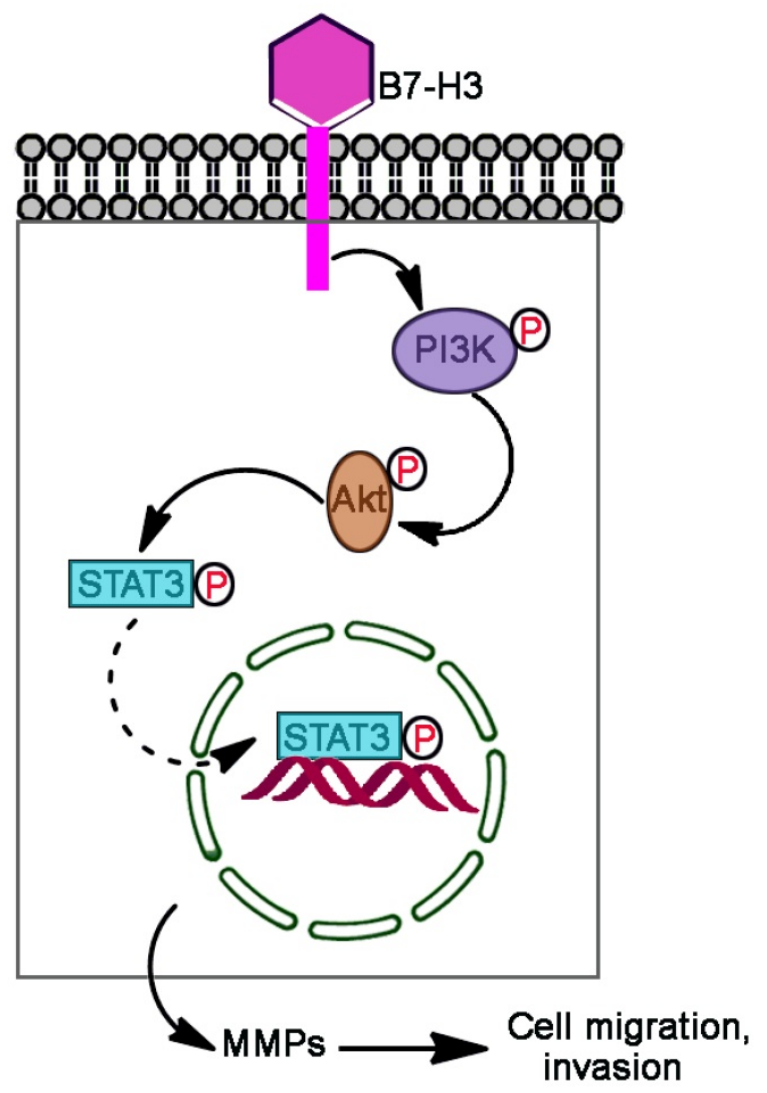

Fig.7. Proposed mechanism by which $\mathrm{B} 7-\mathrm{H} 3$ promotes the migration and invasion of bladder cancer cells. B7-H3 regulates the migration and invasion of bladder cancer cells via the PI3K/Akt/STAT3 signaling pathway.

\section{Conclusion}

In summary, we found that $\mathrm{B} 7-\mathrm{H} 3$ is significantly upregulated in human bladder cancer tissues compared with adjacent normal tissues, overexpression of B7-H3 promoted cell migration and invasion but not proliferation and apoptosis, and knockdown B7-H3 suppressed the expression of MMP2 and MMP9 via the PI3K/Akt/STAT3 signaling pathway (Fig. 7).

\section{Abbreviations}

MMP: matrix metalloproteinase; overB7-H3: B7-H3 overexpression; overNC: negative control overexpression; PD-1: programmed cell death 1; PI: propidium iodide; PI3K: phosphatidylinositol 3-kinase; Q-PCR: quantitative RT-PCR; siB7-H3: B7-H3 siRNA; siNC: negative control siRNA.

\section{Acknowledgments}

This study was supported by a grant from the National Natural Science Foundation of China (No. 
81471862, 31070792), Student's Platform for Innovation and Entrepreneurship Training Program (No. 201590031038).

\section{Competing Interests}

The authors have declared that no competing interest exists.

\section{References}

1. Siegel RL, Miller KD, Jemal A. Cancer statistics, 2016. CA Cancer J Clin. 2016; 66: 7-30.

2. Mellman I, Coukos G, Dranoff G. Cancer immunotherapy comes of age. Nature. 2011; 480: 480-9.

3. Aoun F, Kourie HR, Sideris S, Roumeguere T, Velthoven R, Gil T. Checkpoint inhibitors in bladder and renal cancers: results and perspectives. Immunotherapy. 2015; 7: 1259-71.

4. Kates M, Sopko NA, Matsui H, Drake CG, Hahn NM, Bivalacqua TJ. Immune checkpoint inhibitors: a new frontier in bladder cancer. World J Urol. 2016; 34: 49-55.

5. Li Y, Li F, Jiang F, Lv X, Zhang R, Lu A, et al. A Mini-Review for Cancer Immunotherapy: Molecular Understanding of PD-1/PD-L1 Pathway \&amp; Translational Blockade of Immune Checkpoints. Int J Mol Sci. 2016; 17.

6. Powles T, Eder JP, Fine GD, Braiteh FS, Loriot Y, Cruz C, et al. MPDL3280A (anti-PD-L1) treatment leads to clinical activity in metastatic bladder cancer. Nature. 2014; 515: 558-62.

7. Chapoval AI, Ni J, Lau JS, Wilcox RA, Flies DB, Liu D, et al. B7-H3: a costimulatory molecule for T cell activation and IFN-gamma production. Nat Immunol. 2001; 2: 269-74.

8. Wang L, Kang FB, Shan BE. B7-H3-mediated tumor immunology: Friend or foe? Int J Cancer. 2014; 134: 2764-71.

9. Steinberger P, Majdic O, Derdak SV, Pfistershammer K, Kirchberger S, Klauser C, et al. Molecular characterization of human 4Ig-B7-H3, a member of the B7 family with four Ig-like domains. J Immunol. 2004; 172: 2352-9.

10. Leitner J, Klauser C, Pickl WF, Stockl J, Majdic O, Bardet AF, et al. B7-H3 is a potent inhibitor of human T-cell activation: No evidence for B7-H3 and TREML2 interaction. Eur J Immunol. 2009; 39: 1754-64.

11. Nygren MK, Tekle C, Ingebrigtsen VA, Fodstad O. B7-H3 and its relevance in cancer; immunological and non-immunological perspectives. Front Biosci (Elite Ed). 2011; 3: 989-93.

12. Loos M, Hedderich DM, Ottenhausen M, Giese NA, Laschinger M, Esposito I, et al. Expression of the costimulatory molecule B7-H3 is associated with prolonged survival in human pancreatic cancer. BMC Cancer. 2009; 9: 463.

13. Wu CP, Jiang JT, Tan M, Zhu YB, Ji M, Xu KF, et al. Relationship between co-stimulatory molecule $\mathrm{B} 7-\mathrm{H} 3$ expression and gastric carcinoma histology and prognosis. World J Gastroenterol. 2006; 12: 457-9.

14. Boorjian SA, Sheinin Y, Crispen PL, Farmer SA, Lohse CM, Kuntz SM, et al. T-cell coregulatory molecule expression in urothelial cell carcinoma: clinicopathologic correlations and association with survival. Clin Cancer Res. 2008; $14: 4800-8$

15. Wu D, Zhang Z, Pan H, Fan $Y$, Qu P, Zhou J. Upregulation of the B7/CD28 family member B7-H3 in bladder cancer. Oncol Lett. 2015; 9: 1420-4.

16. Xylinas E, Robinson BD, Kluth LA, Volkmer BG, Hautmann R, Kufer R, et al. Association of T-cell co-regulatory protein expression with clinical outcomes following radical cystectomy for urothelial carcinoma of the bladder. Eur J Surg Oncol. 2014; 40: 121-7.

17. Loo D, Alderson RF, Chen FZ, Huang L, Zhang W, Gorlatov $S$, et al. Development of an Fc-enhanced anti-B7-H3 monoclonal antibody with potent antitumor activity. Clin Cancer Res. 2012; 18: 3834-45.

18. Chen Z, Yang J, Wang G, Song B, Li J, Xu Z. Attenuated expression of xeroderma pigmentosum group $C$ is associated with critical events in human bladder cancer carcinogenesis and progression. Cancer Res. 2007; 67: 4578-85.

19. Yuan F, Xu Z, Yang M, Wei Q, Zhang Y, Yu J, et al. Overexpressed DNA polymerase iota regulated by JNK/c-Jun contributes to hypermutagenesis in bladder cancer. PLoS One. 2013; 8: e69317.

20. Brown GT, Murray GI. Current mechanistic insights into the roles of matrix metalloproteinases in tumour invasion and metastasis. J Pathol. 2015; 237: 273-81.

21. Kang FB, Wang L, Jia HC, Li D, Li HJ, Zhang YG, et al. B7-H3 promotes aggression and invasion of hepatocellular carcinoma by targeting epithelial-to-mesenchymal transition via JAK2/STAT3/Slug signaling pathway. Cancer Cell Int. 2015; 15: 45.

22. Wang J, Chong KK, Nakamura Y, Nguyen L, Huang SK, Kuo C, et al. B7-H3 associated with tumor progression and epigenetic regulatory activity in cutaneous melanoma. J Invest Dermatol. 2013; 133: 2050-8.

23. Li H, Zhang B, Liu Y, Yin C. EBP50 inhibits the migration and invasion of human breast cancer cells via LIMK/cofilin and the PI3K/Akt/mTOR/MMP signaling pathway. Med Oncol. 2014; 31: 162.

24. Niu $H, W u$ B, Peng $Y$, Jiang $H$, Zhang $Y$, Wang J, et al. RNA interference-mediated knockdown of RhoGDI2 induces the migration and invasion of human lung cancer A549 cells via activating the PI3K/Akt pathway. Tumour Biol. 2015; 36: 409-19.

25. Wu X, Chen Y, Li G, Xia L, Gu R, Wen X, et al. Her3 is associated with poor survival of gastric adenocarcinoma: Her3 promotes proliferation, survival and migration of human gastric cancer mediated by PI3K/AKT signaling pathway. Med Oncol. 2014; 31: 903.

26. Chen W, Liu P, Wang Y, Nie W, Li Z, Xu W, et al. Characterization of a soluble B7-H3 (sB7-H3) spliced from the intron and analysis of sB7-H3 in the sera of patients with hepatocellular carcinoma. PLoS One. 2013; 8: e76965.

27. Masuda A, Arai K, Nishihara D, Mizuno T, Yuki H, Kambara T, et al. Clinical significance of serum soluble $\mathrm{T}$ cell regulatory molecules in clear cell renal cell carcinoma. Biomed Res Int. 2014; 2014: 396064.

28. Zhang G, Xu Y, Lu X, Huang H, Zhou Y, Lu B, et al. Diagnosis value of serum B7-H3 expression in non-small cell lung cancer. Lung Cancer. 2009; 66: 245-9.

29. Jacob A, Prekeris R. The regulation of MMP targeting to invadopodia during cancer metastasis. Front Cell Dev Biol. 2015; 3: 4.

30. The Cancer Genome Atlas Research Network. Comprehensive molecular characterization of urothelial bladder carcinoma. Nature. 2014; 507: 315-22

31. Wu X, Obata T, Khan Q, Highshaw RA, De Vere White R, Sweeney C. The phosphatidylinositol-3 kinase pathway regulates bladder cancer cell invasion. BJU Int. 2004; 93: 143-50. 